\title{
Drug Design and Synthesis of First in Class PDZ1 Targeting NHERF1 Inhibitors as Anticancer Agents
}

Antonio Coluccia, ${ }^{\dagger}$ Giuseppe La Regina, ${ }^{\dagger}$ Valentina Naccarato, ${ }^{\dagger}$ Marianna Nalli, ${ }^{\dagger}$ Viviana Orlando, $^{\#}$ Stefano Biagioni, ${ }^{\#}$ Maria Laura De Angelis, ${ }^{\perp}$ Marta Baiocchi, ${ }^{\perp}$ Candice Gautier, ${ }^{\prime}$ Stefano Gianni, ${ }^{\|(\odot)}$ Fiorella Di Pastena, ${ }^{\ddagger}$ Laura Di Magno, ${ }^{\otimes}$ Gianluca Canettieri, ${ }^{\ddagger}$ Addolorata Maria Luce Coluccia, ${ }^{*}, \S$ and Romano Silvestri* ${ }^{\dagger}$ (1)

\footnotetext{
${ }^{\dagger}$ Department of Drug Chemistry and Technologies, Sapienza University of Rome, Laboratory affiliated to Istituto Pasteur Italia Fondazione Cenci Bolognetti, Piazzale Aldo Moro 5, I-00185 Roma, Italy

${ }^{\ddagger}$ Department of Molecular Medicine, Sapienza University of Rome, Laboratory affiliated to Istituto Pasteur Italia - Fondazione Cenci Bolognetti, Viale Regina Elena, 291, I-00161 Roma, Italy

${ }^{\S}$ Department of Biological and Environmental Sciences and Technologies, University of Salento, I-73100 Lecce, Italy

"Department of Biochemistry, Sapienza University of Rome, Laboratory affiliated to Istituto Pasteur Italia - Fondazione Cenci Bolognetti, Piazzale Aldo Moro 5, I-00185 Roma, Italy

${ }^{\perp}$ Department of Oncology and Molecular Medicine, Istituto Superiore di Sanità, Viale Regina Elena, 299, 00161 Rome, Italy

"Department of Biology and Biotechnologies “Charles Darwin”, Sapienza University of Rome, Piazzale Aldo Moro 5, I-00185 Roma, Italy

${ }^{\otimes}$ Center for Life Nano Science@Sapienza, Istituto Italiano di Tecnologia, 00161 Rome, Italy
}

\section{Supporting Information}

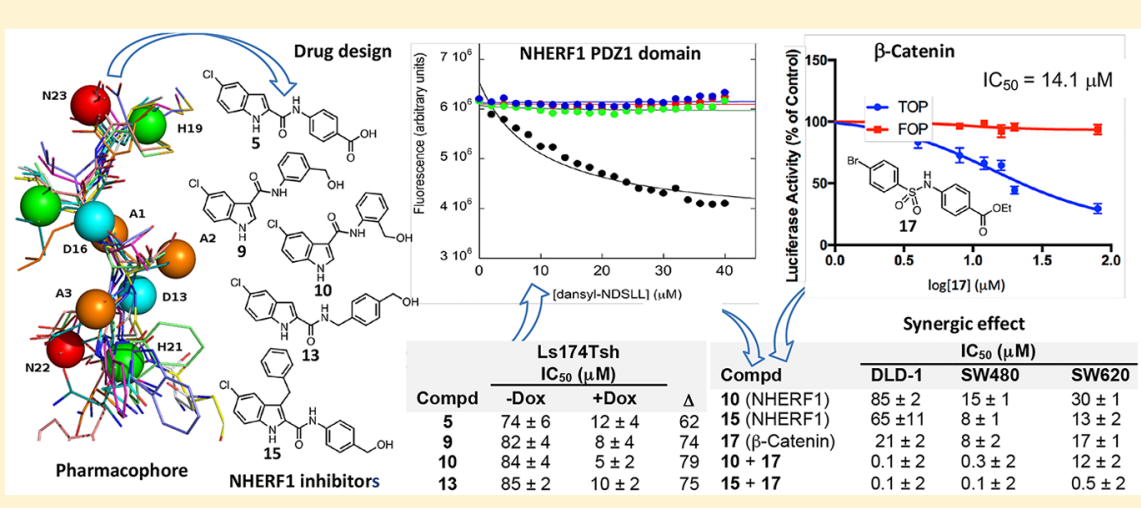

ABSTRACT: Targeted approaches aiming at modulating NHERF1 activity, rather than its overall expression, would be preferred to preserve the normal functions of this versatile protein. We focused our attention on the NHERF1/PDZ1 domain that governs its membrane recruitment/displacement through a transient phosphorylation switch. We herein report the design and synthesis of novel NHERF1 PDZ1 domain inhibitors. These compounds have potential therapeutic value when used in combination with antagonists of $\beta$-catenin to augment apoptotic death of colorectal cancer cells refractory to currently available $\mathrm{Wnt} / \beta$-catenin-targeted agents.

KEYWORDS: cancer, NHERF1, PDZ domain, $\beta$-catenin, drug design, synthesis

NHERF1 $\left(\mathrm{Na}^{+} / \mathrm{H}^{+}\right.$exchanger 3 regulating factor 1$)$ is an integral membrane adaptor protein of 358 amino acids carrying two $\mathrm{NH}_{2}$-terminal PDZ (postsynaptic density 95/discs large/ zona occludens 1) tandem domains. ${ }^{1}$ PDZ1 (11-97 amino acids) and PDZ2 (150-237 amino acids) show 74\% identity to each other and bind to specific carboxyl-terminal motifs on target proteins, such as $\beta$-catenin and PTEN, that may have a pivotal role in tumorigenesis. Oncogenic activity of NHERF1 is strictly dictated by changes on its subcellular localization. ${ }^{1,2}$ While a loss of its physiologic membranous expression usually occurs in early dysplastic human adenomas, especially those of colon and breast, NHERF1 is progressively re-expressed throughout the cytoplasm and nuclei in highly invasive/ metastatic carcinomas. Recent evidence also earmarked

Special Issue: Highlighting Medicinal Chemistry in Italy

Received: November 5, 2018

Accepted: January 14, 2019

Published: January 14, 2019 
NHERF1 as a major driver of a cytoprotective autophagic response elicited by therapeutic inhibition of the Wnt/ $\beta$ Catenin signaling cascade, which triggers colorectal cancers (CRC). ${ }^{3}$ Notably, these studies have started to explore a combined $\beta$-catenin/NHERF1-inhibitory strategy as a fruitful approach to augment apoptotic death of CRC cells refractory to currently available Wnt-targeted agents, particularly at early stages of the disease.

Previous studies focused on small-molecule ligands of the PDZ1-domain of NHERF1 to prevent its ectopic cytoplasmic/ nuclear mislocalization and oncogenic function. ${ }^{4-6}$ These studies predicted the canonical inactivating binding motif for the NHERF1 PDZ1-domain on four D/E-(S/T)-X-(L/V/I/ $\mathrm{M})$ residues that are commonly contained at the $\mathrm{C}$-terminal of its specific ligands, such as DSLL for the $\beta 2$-adrenergic receptor ( $\beta 2$-AR) or ETWM for the parathyroid hormone receptor (PTHR). ${ }^{7}$

The crystal structure of the PDZ1- $\beta 2$-AR C-terminal peptide is available at the protein data bank (PDB code 1GQ4). ${ }^{8}$ The complex was fixed by cutting the chimeric covalent bond between PDZ1 and the substrate (-NDSLL) and submitted to molecular dynamics (MD) simulations to obtain reasonable binding conformations. MD of the complex PDZ1-PTH1R Cterminal peptide (WETVM) was also performed (Figures $1 S$ and 2S, Supporting Information). Trajectory analyses showed that the systems were stable during a simulation time of $50 \mathrm{~ns}$, with an average PDZ1 C $\alpha$ RMSD of 1.66 and $1.73 \AA$ for PDZ1-NDSLL-COOH and PDZ1-WETVM-COOH, respectively. The main interactions between the PDZ1 and the $-\beta 2$ AR substrate observed at the crystal structures were retained during the MD simulations (Supporting Information). The trajectories were clustered by the RMSDs of the binding site residues, giving five clusters for the two studied complexes, each containing representative structures. The obtained 10 structures guided the design of a pharmacophore model (Figure 3S, Supporting Information) that was used to filter out an in-house training set of about 6000 compounds. One out of six selected compounds showed significant NHERF1 inhibition. $^{3}$ We herein report the design and synthesis of new NHERF1 inhibitors 1-14. In preliminary docking studies, these compounds matched the requirements of the pharmacophore model, in particular, the interactions at the $\mathrm{t}-\mathrm{COOH}$ binding pocket. ${ }^{9}$

Compounds 1-14 were synthesized starting from 5-chloro$1 \mathrm{H}$-indole-2-carboxylic acid (18) or its 3-isomer 19 (Scheme 1, Supporting Information). The acids were transformed into the corresponding acyl chlorides with thionyl chloride and coupled with an appropriate aminobenzoate in dichloromethane in the presence of pyridine to afford carboxamides 20-24. Hydrolysis of the ester functionality of 20-24 was performed with lithium hydroxide monohydrate in tetrahydrofuran (5 and 6) or with $3 \mathrm{~N}$ sodium hydroxide in ethanol at $25^{\circ} \mathrm{C}(7,11$, and 12). Alcohol derivatives 1, 3, 4, and 8-10 were obtained by tetrabutylammonium fluoride cleavage in tetrahydrofuran at $25{ }^{\circ} \mathrm{C}$ of the corresponding tertbutyldimethylsilyl derivatives 25-30. The latter compounds were prepared by reaction of 18 or 19 with tertbutyldimethylsilyloxymethylanilines 31-33. Carboxamides 2, 13, 25-30, and 34 were synthesized from the corresponding anilines in the presence of tripyrrolidinophosphonium hexafluorophosphate (PyBOP) and triethylamine in DMF at $25{ }^{\circ} \mathrm{C}$. Compound 14 was obtained after lithium hydroxide of the intermediate methyl tyrosinate 34 .
The ability of $\mathbf{1 - 1 4}$ to inhibit NHERF1 was assessed in Ls174T CRC cells harboring a doxycycline hyclate (Dox)inducible small hairpin RNA (shRNA) for $\beta$-catenin (Ls174Tsh $\beta$-Cat) (Table 1). Our published data indicated

Table 1. Structure and Activity of Compounds 1-14 in Ls174Tsh $\beta$ Cat Cells Expressing Low (-DOX) or High $(+\mathrm{DOX})$ Levels of the Protein Target NHERF1 ${ }^{a}$

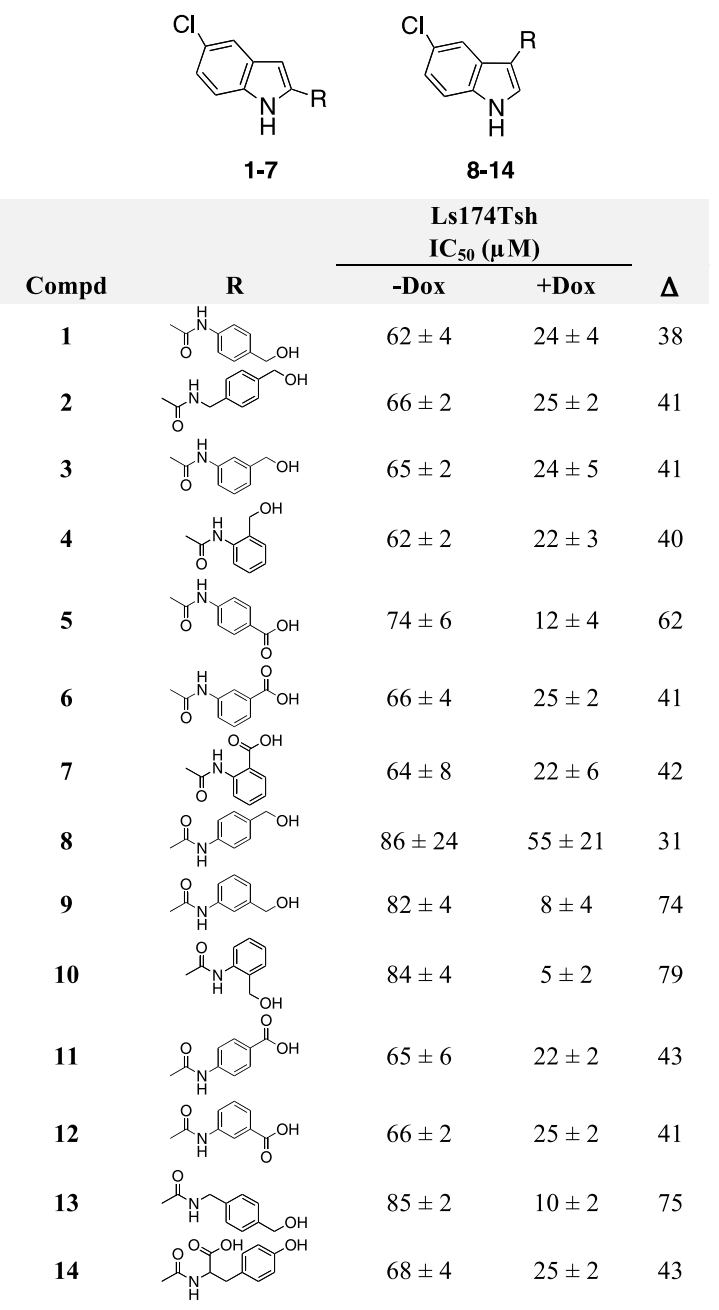

${ }^{a}$ Ls174T cells stably transfected with Dox-inducible shRNA for $\beta$ catenin (Ls174Tsh $\beta$-Cat) were cultured without or with $2 \mu \mathrm{g} / \mathrm{mL}$ of Dox $(-$ Dox $/+$ Dox $)$ for 1,3 , or 5 days.

that such CRC cells exhibited low/undetectable NHERF1 levels at the baseline (i.e., in the absence of Dox), while they markedly increase the expression of the protein as well as a NHERF1-mediated survival response under Dox treatment. ${ }^{3}$ The relative activity of $\mathbf{1 - 1 4}$ can be explained by $\Delta$ values obtained by subtracting the $\mathrm{IC}_{50}$ values of the $\mathrm{Ls} 174 \mathrm{~T} \operatorname{sh} \beta$-Cat cells cultured in the presence or in the absence of Dox. Indeed, NHERF1/PDZ-1 ligand inhibitors are expected to exert their growth-inhibitory effects only on $\beta$-catenin-depleted CRC cells, which highly re-expressed the protein target NHERF1, thus showing the highest $\Delta$ values with regard to Doxuntreated cells. Introduction of a methylene spacer between the 2-carboxamide function and the phenyl group of 1 (-Dox $\mathrm{IC}_{50}=62 \mu \mathrm{M} ;+$ Dox $\left.\mathrm{IC}_{50}=24 \mu \mathrm{M}\right)$ to afford 4 gave a slight improvement of activity. Moving the hydroxymethyl group of 1 from para to meta (3) or ortho (4) only minimally affected the 
difference $(\Delta)$ of activity between the two cell lines. Replacement of the hydroxymethyl of $\mathbf{1}$ with a carboxylic acid function (5) (-Dox $\mathrm{IC}_{50}=74 \mu \mathrm{M} ;+$ Dox $\left.\mathrm{IC}_{50}=12 \mu \mathrm{M}\right)$ improved the $\Delta$ value from 38 to 62 , while the corresponding meta (6) or ortho (7) carboxylic acids gave similar results for 2-4. We synthesized the corresponding analogues 8-14 at position 3 of the indole. Derivatives $9\left(-\right.$ Dox $\mathrm{IC}_{50}=82 \mu \mathrm{M}$; + Dox $\left.\mathrm{IC}_{50}=8 \mu \mathrm{M}\right)$ and $10\left(-\right.$ Dox $\mathrm{IC}_{50}=84 \mu \mathrm{M}$; +Dox $\mathrm{IC}_{50}$ $=5 \mu \mathrm{M})$ bearing the hydroxymethyl at positions meta and ortho ( $\Delta$ of 74 and 79 , respectively) were superior to the para analogue 7 as well as to the carboxylic acids 11 and 12. The 3indolecarboxamide $13\left(-\right.$ Dox $\mathrm{IC}_{50}=85 \mu \mathrm{M}$; +Dox $\mathrm{IC}_{50}=10$ $\mu \mathrm{M})$ yielded a good $\Delta$ value of 75 that was 1.8 -fold superior to the isomeric derivative 1 . In sum, compounds 5, 9, 10, and 13 exhibited a remarkable cytotoxicity and the highest $\Delta$ values on Ls174Tsh $\beta$-Cat, which highly re-express the specific protein target NHERF1.

The indole $\mathrm{NH}$ and the carboxamide oxygen formed $\mathrm{H}$ bonds with the Leu27 backbone. Hydrophobic contacts were observed between the phenyl ring and Tyr24, Phe26, and Ile78 as well as between the indole ring and Val75 and Val76. It is noteworthy that the carboxylic group of derivatives 5-7 formed polar contacts with the Arg80 (Figure 1, top panel). Derivatives 8-14 showed binding modes with different patterns in H-bonding. In comparing the alcohol 9 and its corresponding carboxylic acid 12, the indole $\mathrm{NH}$ formed an $\mathrm{H}$ bond with the His 72 backbone, the amide oxygen atom formed an $\mathrm{H}$-bond with the His27 side chain, the indole ring established hydrophobic contacts with Leu28 and Val75, the phenyl ring arranged hydrophobic contacts with Tyr24 and Phe26, and a $\pi$-cation interaction with the guanidine moiety of Arg80 occurred. The alcohol and the carboxyl moieties were involved in $\mathrm{H}$-bond/polar contact with the terminal $\mathrm{COOH}$ binding pocket (Figure 1, bottom panel).

The binding of $9, \mathbf{1 0}$, and 13 to NHERF1 PDZ1 was assessed by means of a dansylated peptide corresponding to the C-terminal sequence of $\beta 2$-AR (DNDSLL) using a a fluorescent pseudowild type produced by replacing Tyr38 of PDZ1 with a Trp. 9 The presence of a constant concentration of 9,10 , or 13 abolished the binding C-terminal sequence of $\beta 2$-AR with an affinity of about $10 \mu \mathrm{M}$, thus validating these compounds as specific inhibitors of the NHERF1/PDZ1 domain (Figure 2). To further test the ability of $\mathbf{9 , 1 0}$, and $\mathbf{1 3}$ to bind NHERF1 PDZ1, we performed urea-induced denaturation in the presence of the inhibitors. In fact, the ability to bind the native, rather than the denatured, state of the protein induces a stabilization of the domain. Such a stabilization is correlated to the affinity of the compounds to PDZ1 NHERF1 (Figure 4S, Supporting Information). The results provide compelling evidence of the inhibition of NHERF1 PDZ1 by derivatives 9, 10, and 13.

Derivatives 5, 9, 10, and 13 with the highest $\Delta$ values as inhibitors of Ls174Tsh $\beta$-Cat cells were also tested in three CRC cell lines, such as DLD-1, SW480, and SW620. It is known that NHERF1 is expressed at detectable baseline levels in SW480 and SW620 but not in DLD-1 cells. ${ }^{3}$ This relatively explains why DLD-1 cells showed the highest $\mathrm{IC}_{50}$ values compared to SW480 and SW620 cells (Table 2) at micromolar concentrations quite similar to those previously obtained in Dox-untreated Ls174Tsh $\beta$-Cat cells (Table 1) (additional cancer cell lines are reported in Table 1S, Supporting Information).

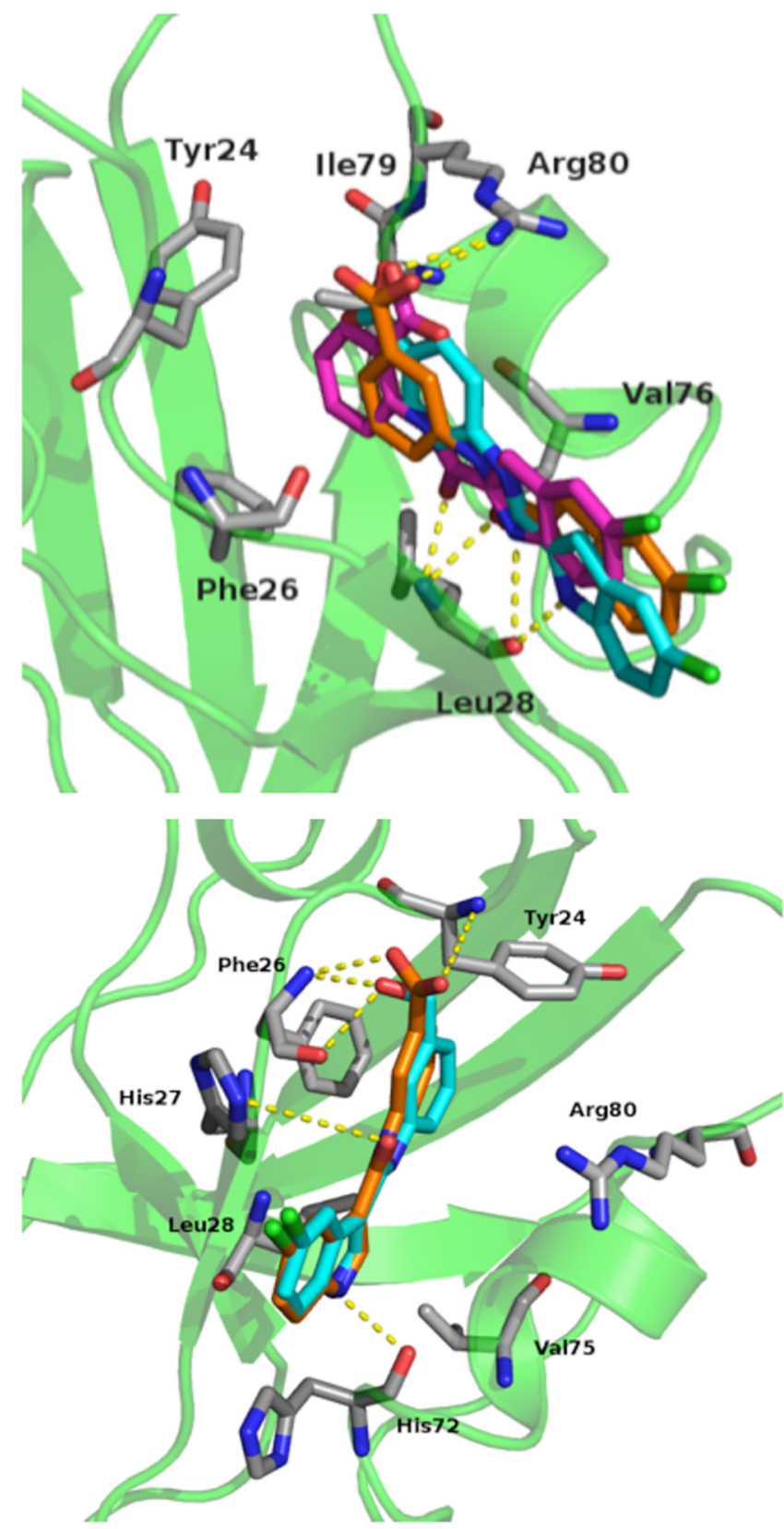

Figure 1. Proposed binding modes. Top panel: derivatives 5 (cyan), 6 (orange), and 7 (magenta). Bottom panel: derivatives 9 (cyan) and 12 (orange). Residues involved in interactions are reported as stick. PDZ1 is reported as cartoon (green). The H-bonds are depicted as yellow dotted lines.

We previously reported that 3-benzyl-5-chloro- $N$-(4(hydroxymethyl)phenyl)-1H-indole-2-carboxamide (15), a NHERF1 PDZ1 inhibitor with - Dox $\mathrm{IC}_{50}=52 \mu \mathrm{M}$ and + Dox $\mathrm{IC}_{50}=8 \mu \mathrm{M}$ synergized $N$-(2-methyl-4-nitrophenyl)-2,5dichlorobenzenesulfonamide (FH535, 16) and the pyrvinium pamoate inhibitor of $\beta$-catenin-dependent transcription in triggering massive apoptosis of Ls174T and DLD1 CRC cells, thereby preventing persistent ERK1/2 phospho-activation mediated by single $\beta$-Catenin targeting., ${ }^{3,11}$ We therefore speculated the potential benefit of a synergic activity of NHERF1 PDZ1 and $\beta$-catenin inhibitors in CRC therapy.

We compared 10, the compound with the highest $\Delta$ value within this series, and $\mathbf{1 5},^{3}$ with two $\beta$-Catenin inhibitors, FH535 (16) $)^{12}$ and 17 (Chart 1S, Supporting Information). We 


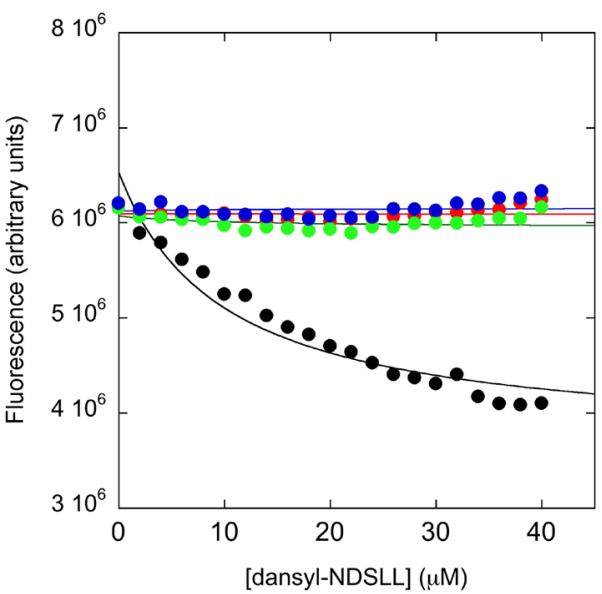

Figure 2. Binding of PDZ1 NHERF1 Y38W to the ligand dansylNDSLL in the presence (black circles) and in the absence of $5 \mu \mathrm{M}$ of 9 (green), 10 (red), or 13 (blue). Fluorescence data were recorded in the presence of $50 \mathrm{mM}$ Na phosphate $\mathrm{pH} 7.2,300 \mathrm{mM} \mathrm{NaCl}, 5 \mathrm{mM}$ DTT, $20 \%$ DMSO, at $25{ }^{\circ} \mathrm{C}$. Lines are the best fit to a hyperbolic binding transition. The binding between PDZ1 NHERF1 Y38W to the ligand dansyl-NDSLL was abolished in the presence of $\mathbf{9 , 1 0}$, or 13.

Table 2. Cell Growth Inhibition of DLD-1, SW480, and SW620 Cell Lines by Compounds 5, 9, 10, and $13^{a}$

\begin{tabular}{cccc} 
& & \multicolumn{2}{c}{$\mathrm{IC}_{50}(\mu \mathrm{M})$} \\
\cline { 3 - 4 } compd & $\mathrm{DLD}-1$ & $\mathrm{SW} 480$ & $\mathrm{SW620}$ \\
$\mathbf{5}$ & $68 \pm 1$ & $17 \pm 1$ & $22 \pm 1$ \\
$\mathbf{9}$ & $82 \pm 2$ & $13 \pm 1$ & $27 \pm 1$ \\
$\mathbf{1 0}$ & $85 \pm 2$ & $15 \pm 1$ & $30 \pm 1$ \\
$\mathbf{1 3}$ & $84 \pm 2$ & $16 \pm 1$ & $31 \pm 1$
\end{tabular}

${ }^{a}$ Cytotoxic concentrations for the indicated cell lines. Experiments were performed in triplicate and repeated at least twice.

tested the effect of increasing concentrations of $\mathbf{1 7}$ on the Wnt signaling pathway by performing Wnt reporter assays in 293T cells. We transfected TOPFlash (TOP) reporter plasmid, containing eight repeats of TCF/LEF binding site or FOPFlash (FOP) plasmid, where the TFC/LEF binding sites were mutated as negative control. ${ }^{13}$ The Wnt pathway was activated using the GSK3beta inhibitor LiCl. Compound 17 inhibited the activity of the TOP reporter, with an $\mathrm{IC}_{50}$ of 14.1 $\mu \mathrm{M}$ (Figure 5S, Supporting Information). Conversely, this compound failed to prevent the activity of FOP reporter, indicating the specificity of the inhibition of the Wnt pathway.

Indeed, a $1: 1$ combination of a NHERF1 inhibitor with a $\beta$ catenin inhibitor significantly improved the growth inhibition of all CRC cells. All combinations were superior to each single tested compound in DLD-1 and SW480 cells. Compound combinations $10+17,15+16$, and $15+17$ inhibited the SW480 cells at submicromolar concentrations, and the most potent combination was $15+17\left(\mathrm{IC}_{50}=0.1 \mu \mathrm{M}\right)$. Combinations $10+17$ and $15+17$ showed the higher effectiveness, and $15+17$ was the most potent with $\mathrm{IC}_{50}$ values of 0.1 and $0.5 \mu \mathrm{M}$ (Table 3 ).

Cancer stem cells (CSCs) have received growing interest during the last years because they have proved to be selectively resistant to chemotherapics, thus giving an explanation to the relapse that in most cases follows fast tumor shrinkage induced by standard therapies. In this frame, the identification of
Table 3. Synergistic Cell Growth Inhibition of SW480 and SW620 Cell Lines by Compounds 10, 15, 16, and $17^{a, b}$

\begin{tabular}{lllrl} 
& & \multicolumn{3}{c}{$\mathrm{IC}_{50}(\mu \mathrm{M})$} \\
\cline { 3 - 5 } compd & inhibitor & DLD-1 & SW480 & SW620 \\
$\mathbf{1 0}$ & NHERF1 & $85 \pm 2$ & $15 \pm 1$ & $30 \pm 1$ \\
$\mathbf{1 5}$ & NHERF1 & $65 \pm 11$ & $8 \pm 1$ & $13 \pm 2$ \\
$\mathbf{1 6}$ & $\beta$-Cat & $28 \pm 5$ & $10 \pm 2$ & $19 \pm 1$ \\
$\mathbf{1 7}$ & $\beta$-Cat & $21 \pm 2$ & $8 \pm 2$ & $17 \pm 1$ \\
$\mathbf{1 0}+\mathbf{1 6}$ & NHERF1 + $\beta$-Cat & $0.8 \pm 2$ & $3.0 \pm 2$ & $22 \pm 2$ \\
$\mathbf{1 0}+\mathbf{1 7}$ & NHERF1 + $\beta$-Cat & $0.1 \pm 2$ & $0.3 \pm 2$ & $12 \pm 2$ \\
$\mathbf{1 5}+\mathbf{1 6}$ & NHERF1 + $\beta$-Cat & $0.5 \pm 3$ & $0.9 \pm 2$ & $18 \pm 1$ \\
$\mathbf{1 5}+\mathbf{1 7}$ & NHERF1 + $\beta$-Cat & $0.1 \pm 2$ & $0.1 \pm 2$ & $0.5 \pm 2$
\end{tabular}

${ }^{a}$ Cytotoxic concentrations for the indicated cell lines. Experiments were performed in triplicate repeated at least twice. ${ }^{b}$ Compound combinations were in a $1: 1$ ratio.

targeted drugs active on CSCs may give hope of full tumor eradication. Preliminary screening of two APC-mutated CSCs indicated that their sensitivity to compounds 10 and 17 was broadly comparable to that of standard cell lines (Table 4). Analysis on a wider panel of CSCs is ongoing in order to verify a possible role of different mutational profiles of individual CSCs on drug sensitivity.

Table 4. Cell Growth Inhibition of CSC13 and CSC8 Cancer Stem Cell Lines by Compounds 10 and $17^{a}$

\begin{tabular}{clrr} 
& & \multicolumn{2}{c}{$\mathrm{IC}_{50}(\mu \mathrm{M})$} \\
\cline { 3 - 4 } compd & inhibitor & CSC13 & CSC8 \\
10 & NHERF1 & $12 \pm 1$ & $8 \pm 1$ \\
17 & $\beta$-Cat & $22 \pm 2$ & $13 \pm 2$
\end{tabular}

${ }^{a}$ Cytotoxic concentrations for the indicated CSCs. Experiments were performed in triplicate repeated at least twice.

Since physiological or pathological roles of NHERF1 are related to its subcellular localization, targeted approaches aiming at modulating NHERF1 activity, rather than its overall expression, would be preferred to preserve the normal functions of this versatile protein. By far, particular attention has been paid to the NHERF1/PDZ1-domain that governs its membrane recruitment/displacement through a transient phosphorylation switch. We herein characterized novel NHERF1 PDZ1 domain inhibitors with therapeutic value when used in combination with antagonists of $\beta$-catenin. This double-targeted strategy shows potential to augment apoptotic death of CRC cells refractory to currently available Wnttargeted agents, particularly at early stages of the disease.

\section{ASSOCIATED CONTENT}

\section{Supporting Information}

The Supporting Information is available free of charge on the ACS Publications website at DOI: 10.1021/acsmedchemlett.8b00532.

Additional information for MD simulations and binding modes, experimental procedures of synthesis, computational studies, NHERF1 PDZ1 binding studies, Wnt reporter assay, cell cultures and biological assays, and additional cancer cell growth inhibition (PDF) 


\section{AUTHOR INFORMATION}

\section{Corresponding Authors}

*(R.S.): Phone: +39 064991 3800. Fax: +39 064991 3133. E-

mail: romano.silvestri@uniroma1.it.

*(A.M.L.C.): Phone: +390836 63669. Fax: +390836 63669 .

E-mail: malu.coluccia@unisalento.it.

\section{ORCID}

Antonio Coluccia: 0000-0002-7940-8206

Giuseppe La Regina: 0000-0003-3252-1161

Stefano Gianni: 0000-0003-1653-1925

Romano Silvestri: 0000-0003-2489-0178

\section{Author Contributions}

Chemistry: A.C., computational studies; G.L.R., V.N., and M.N., chemical synthesis. Biology: V.O., cancer cells; M.B., cancer stem cells; C.G. and S.G., PDZ domain; F.D.P., L.D.M., and G.C., $\beta$-catenin; A.M.L.C., NHERF1.

\section{Funding}

We gratefully acknowledge funding from Italian PRIN 2015 (2015FCHJ8E to R.S.), Sapienza University 2017 (RP11715C7D1CF0D1 to G.L.R.), AIRC-IG (14236 to A.M.L.C. and 17575 to G.C.), AFM-Telethon 21025 and Institute Pasteur Italy (to G.C.), and the European Union's Horizon 2020 research and innovation program under Marie Sklodowska-Curie Grant Agreement No. 675341 (to S.G).

\section{Notes}

The authors declare no competing financial interest.

\section{ACKNOWLEDGMENTS}

M50 Super 8x TOPFlash and FOPFlah plasmids were a gift from Randall Moon (Addgene plasmid no. 12456).

\section{ABBREVIATIONS}

NHERF1, $\mathrm{Na}^{+} / \mathrm{H}^{+}$exchanger 3 regulating factor 1 ; PDZ, postsynaptic density 95/discs large/zona occludens 1; CRC, colorectal cancer; Dox, doxycycline

\section{REFERENCES}

(1) Vaquero, J.; Nguyen Ho-Bouldoires, T. H.; Claperon, A.; Fouassier, L. Role of the PDZ scaffold protein NHERF1/EBP50 in cancer biology: from signaling regulation to clinical relevance. Oncogene 2017, 36, 3067-3079.

(2) Georgescu, M. M.; Morales, F. C.; Molina, J. R.; Hayashi, Y. Roles of NHERF1/EBP50 in cancer. Curr. Mol. Med. 2008, 8, 459468.

(3) Saponaro, C.; Sergio, S.; Coluccia, A.; De Luca, M.; La Regina, G.; Mologni, L.; Famiglini, V.; Naccarato, V.; Bonetti, D.; Gautier, C.; Gianni, S.; Vergara, D.; Salzet, M.; Fournier, I.; Bucci, C.; Silvestri, R.; Gambacorti Passerini, C.; Maffia, M.; Coluccia, A. M. L. AML. $\beta$ Catenin knockdown promotes NHERF1-mediated survival of colorectal cancer cells: implications for a double-targeted therapy. Oncogene 2018, 37, 3301-3316.

(4) Mayasundari, A.; Ferreira, A. M.; He, L.; Mahindroo, M.; Bashford, D.; Fujii, N. Rational design of the first small-molecule antagonists of NHERF1/EBP50 PDZ domains. Bioorg. Med. Chem. Lett. 2008, 18, 942-945.

(5) Du, G.; Gu, Y.; Hao, C.; Yuan, Z.; He, J.; Jiang, W. G.; et al. The cellular distribution of $\mathrm{Na}^{+} / \mathrm{H}^{+}$exchanger regulatory factor 1 is determined by the PDZ-I domain and regulates the malignant progression of breast cancer. Oncotarget 2016, 7, 29440-29453.

(6) Fitzpatrick, J. M.; Pellegrini, M.; Cushing, P. R.; Mierke, D. F. Small molecule inhibition of $\mathrm{Na}^{+} / \mathrm{H}^{+}$exchange regulatory factor 1 and parathyroid hormone 1 receptor interaction. Biochemistry 2014, 53, 5916-5922.
(7) Mamonova, T.; Kurnikova, M.; Friedman, P. A. Structural basis for NHERF1 PDZ domain binding. Biochemistry 2012, 51, 31103120.

(8) Karthikeyan, S.; Leung, T.; Ladias, J. A. A. Structural determinants of the $\mathrm{Na}^{+} / \mathrm{H}^{+}$exchanger regulatory factor interaction with the beta 2 adrenergic and platelet-derived growth factor receptors. J. Biol. Chem. 2002, 277, 18973-18987.

(9) Gianni, S.; Engström, A.; Larsson, M.; Calosci, N.; Malatesta, F.; Eklund, L.; Ngang, C. C.; Travaglini-Allocatelli, C.; Jemth, P. The kinetics of PDZ domain-ligand interactions and implications for the binding mechanism. J. Biol. Chem. 2005, 280, 34805-34812.

(10) Handeli, S.; Simon, J. A. A small-molecule inhibitor of Tcf/ beta-catenin signaling down-regualtes PPAR $\gamma$ and PPAR $\delta$ activities. Mol. Cancer Ther. 2008, 7, 521-529.

(11) Thorne, C. A.; Hanson, A. J.; Schneider, J.; Tahinci, E.; Orton, D.; Cselenyi, C. S.; Jernigan, K. K.; Meyers, K. C.; Hang, B. I.; Waterson, A. G.; Kim, K.; Melancon, B.; Ghidu, V. P.; Sulikowski, G. A.; La Fleur, B.; Salic, A.; Lee, L. A.; Miller, D. M., III; Lee, E. Smallmolecule inhibition of Wnt signaling through activation of casein kinase $1 \alpha$. Nat. Chem. Biol. 2010, 6, 829-836.

(12) Chen, Y.; Rao, X.; Huang, K.; Jiang, X.; Wang, H.; Teng, L. FH535 inhibits proliferation and motility of colon cancer cells by targeting Wnt/ $\beta$-catenin signaling pathway. J. Cancer 2017, 8, 31423153.

(13) Veeman, M. T.; Slusarski, D. C.; Kaykas, A.; Louie, S. H.; Moon, R. T. Zebrafish prickle, a modulator of noncanonical Wnt/Fz signaling, regulates gastrulation movements. Curr. Biol. 2003, 13, 680-685. 